\title{
AN INTERMEDIATE THEORY FOR A PURELY INSEPARABLE GALOIS THEORY(1)
}

\author{
BY \\ JAMES K. DEVENEY
}

\begin{abstract}
Let $K$ be a finite dimensional purely inseparable modular extension of $F$, and let $L$ be an intermediate field. This paper is concerned with an intermediate theory for the Galois theory of purely inseparable extensions using higher derivations [4]. If $L$ is a Galois intermediate field and $M$ is the field of constants of all higher derivations on $L$ over $F$, we prove that every higher derivation on $L$ over $F$ extends to $K$ if and only if $K=L \otimes_{M} J$ for some field $J$. Similar to classical Galois theory the distinguished intermediate fields are those which are left invariant under a standard generating set for the group of all rank $t$ higher derivations on $K$ over $F$. We prove: $L$ is distinguished if and only if $L$ is $M$-homogeneous (4.9).
\end{abstract}

I. Introduction. This paper is concerned with an intermediate theory for the Galois theory of purely inseparable extensions using higher derivations [4]. In classical Galois theory, if $G$ is a full group of automorphisms on a field $C$ with field of constants $E$, then an intermediate field $D$ is distinguished if and only if it is invariant under $G$. Moreover, all automorphisms on $D$ over $E$ can be extended to $C$. Let $K$ be a finite dimensional purely inseparable modular extension of $F$, and let $L$ be an intermediate field. We prove: (1) the only intermediate fields invariant under all higher derivations on $K$ over $F$ are of the form $F\left(K^{p^{n}}\right)$ for some $n$; (2) if $L$ is a Galois intermediate field (i.e., the field of constants of a group of higher derivations on $K$ over $F$ ) and $M$ is the field of constants of all higher derivations on $L$ over $F$, then every higher derivation on $L$ over $F$ extends to $K$ if and only if $K=L \otimes_{M} J$ for some field $J$. Combining (1) and (2) shows that the only intermediate fields with properties completely analogous to the classical case are $K$ and $F$. Considering the Dedekind independence theorem for automorphisms and [7, Theorem 19, p. 186] a natural alternative is to define the distinguished intermediate fields to be those which are left invariant under a standard generating set for the group of all higher derivations on $K$ over $F$. If $L$ is a distinguished intermediate field, then $K$ is modular over $L$ and $L$ is modular over $F$. We provide an example which shows the converse does not hold. Two conditions equivalent to being distinguished are established: (1) There exists a subbase $\left\{x_{1}, \ldots, x_{n}\right\}$ for $K$ over $F$ such that $\left\{x_{i}^{p_{i}^{e}}, \ldots, x_{n}^{p_{n}^{e}}\right\}$ is a subbase for $L$ over

Presented to the Society, November 16, 1973; received by the editors June 27, 1973.

AMS (MOS) subject classifications (1970). Primary 12F15.

Key words and phrases. Higher derivation, iterative higher derivation, dual basis, $M$-homogeneous intermediate fields.

(1)This work was supported in part by the Committee on Faculty Research Support administered by Dr. Nickolas Heerema. 
$F$ for some $e_{1}, \ldots, e_{n}$; (2) There exists $T=T_{1} \cup \cdots \cup T_{n}$ a subbase for $K$ over $F$, the elements of $T_{i}$ being of exponent $i$ over $F$, such that $L=L \cap F\left(T_{1}\right) \otimes \cdots$ $\otimes L \cap F\left(T_{n}\right)$ and $F\left(T_{i}\right)$ is modular over $L \cap F\left(T_{i}\right)$ for all $i$.

II. Definitions and preliminary results. Throughout this paper, $K$ will be a field of characteristic $p \neq 0,2$. A rank $t$ higher derivation on $K$ is a sequence $d$ $=\left\{d_{i} \mid 0 \leq i<t+1\right\}$ of additive maps of $K$ into $K$ such that $d_{r}(a b)$ $=\sum\left\{d_{i}(a) d_{j}(b) \mid i+j=r\right\}$ and $d_{0}$ is the identity map. The set $H^{t}(K)$ of all rank $t$ higher derivations on $K$ is a group with respect to the composition $d \circ e=f$ where $f_{j}=\sum\left\{d_{m} e_{n} \mid m+n=j\right\}[1$, Theorem 1, p. 33]. The field of constants of a subset $G \subseteq H^{t}(K)$ is $\left\{a \in K \mid d_{i}(a)=0, i>0,\left(d_{i}\right) \in G\right\}$. $H_{F}^{t}(K)$ will denote the group of all rank $t$ higher derivations on $K$ whose field of constants contains the subfield $F$.

(2.1) [2, Theorem 1]. Let $B$ be a $p$-basis for $K$ and let $f: Z \times B \rightarrow K$ be an arbitrary function. There is a unique $\left(d_{i}\right) \in H^{\infty}(K)$ such that for each $b \in B$ and $i \in Z, d_{i}(b)=f(i, b)$.

A higher derivation $d$ in $H^{\infty}(K)$ is called iterative of index $q$, or simply iterative, if $\left(\begin{array}{l}i \\ j\end{array}\right) d_{q i}=d_{q i} d_{q(i-j)}$ for all $i$ and $j \leq i$, whereas $d_{m}=0$ if $q \nmid m$. If $d \in H^{\infty}(K)$ is iterative of index $q$, and $a$ is in $K$, then $a d=e$ where $e_{q i}=a^{i} d_{q i}$, and $e_{j}=0$ if $q \nmid j$. It is clear that $a d$ is a higher derivation. A finite rank higher derivation $(t<\infty)$ is iterative if it is the first $t+1$ maps of an infinite iterative higher derivation. Given $d \in H_{F}^{t}(K)$ of index $q, V(d)=e \in H_{F}^{t}(K)$ where $e_{(q+1) i}=d_{q i}$ for $(q+1) i \leq t$ and $e_{j}=0$ if $(q+1) \nmid j, j \leq t$.

Throughout the remainder of this paper, $K$ will be a finite dimensional purely inseparable modular extension of $F$ of exponent $n$, and $p^{n-1} \leq t<\infty$. Since $K$ is modular over $F, K=F\left(x_{1}\right) \otimes \cdots \otimes F\left(x_{n}\right)$. Any such elements $x_{1}, \ldots, x_{n}$ is called a subbase for $K$ over $F$.

(2.2) [3, p. 436]. Let $\left(d_{i}\right) \in H^{t}(K)$ and $a \in K$. Then $d_{i p}\left(a^{p}\right)=\left(d_{i}(a)\right)^{p}$ and if $p$ and $j$ are relatively prime, then $d_{j}\left(a^{p}\right)=0$.

(2.3) [4, Lemma 3.7]. Let $K$ be a purely inseparable modular extension of $F$, and let $N$ be a subbase for $K$ over $F$. Then there exists a subset $S$ of $F$ such that $N \cup S$ is a $p$-basis for $K$.

(2.4) Definition. Let $\left\{x_{1,1}, \ldots, x_{1, j_{1}}, \ldots x_{n, 1}, \ldots, x_{n, j_{n}}\right\}$ be a subbase for $K$ over $F$ where $x_{i, e}$ is of exponent $i$ over $F$. Let $A=\left\{d^{i, e} \mid 1 \leq i \leq n, 1 \leq e \leq j_{i}\right\}$ be the set of rank $t$ higher derivations on $K$ over $F$ defined by

$$
d_{\left[i / p^{i}\right]+1}^{i, e}\left(x_{r, s}\right)=\delta_{((i, e),(r, s))},
$$

where $\left[t / p^{i}\right]$ is the greatest integer less than or equal to $t / p^{i}$.

$$
d_{\alpha}^{i, e}\left(x_{r, s}\right)=0, \quad 1 \leq i, r \leq n, 1 \leq e \leq j_{i}, \quad 1 \leq s \leq j_{r}, \alpha \neq\left[t / p^{i}\right]+1 .
$$

Then $A$ is a standard set of generators for $H_{F}^{t}(K)$ and $\left\{x_{i, e} \mid 1 \leq i \leq n, 1 \leq e\right.$ $\left.\leq j_{i}\right\}$ is called a dual base for $A$.

For later use, we now list some properties of $A$ which follow from [4, §VI]. Let 
the first nonzero map (of subscript $>0$ ) of $d^{i, e}$ be $d_{z_{i, e^{*}}}^{i, e}$

(2.5) Observations. (a) $A$ is abelian, i.e., all maps which appear in elements of $A$ commute, and each $d^{i, e}$ is iterative of index $z_{i, e}$.

(b) $\left\{x_{r+1,1}^{p^{\prime}}, \ldots, x_{n, j_{n}}^{p^{\prime}}\right\}$ is a subbase for $F\left(K^{p^{\prime}}\right)$ over $F$.

$$
\left\{\left.d_{z_{r+1,1} P^{p^{\prime}}}^{r+1,1}\right|_{F\left(K p^{\prime}\right)}, \ldots,\left.d_{z_{n, \mu_{n}} p^{\prime}}^{n, j_{n}}\right|_{F\left(K p^{\prime}\right)}\right\}
$$

is a vector space basis over $F\left(K^{p^{\prime}}\right)$ for the space of all derivations on $F\left(K^{p^{\prime}}\right)$ over $F\left(K^{p+1}\right)$ and hence these maps have field of constants $F\left(K^{p+1}\right)$.

(d) $d_{z_{i, \rho} p^{\prime}}^{i, e}\left(x p_{k, s}^{\prime}\right)=\delta_{((i, e),(k, s))}, \quad r+1 \leq i, k \leq n, 1 \leq e \leq j_{i}, 1 \leq s \leq j_{k}$.

\section{Invariant subfields and extensions of higher derivations.}

(3.1) Theorem. Let $L$ be a subfield of $K$ containing $F$. Then $L$ is invariant under $H_{F}^{t}(K)$ if and only if $L=F\left(K^{p^{r}}\right)$ for some nonnegative integer $r$.

Proof. Assume $L=F\left(K^{p^{\prime}}\right)$, and let $\left(d_{i}\right) \in H_{F}^{t}(K)$. If $x \in L$, then

$$
x=\sum\left\{a_{i} b p^{p^{\prime}} \mid a_{i} \in F, b_{i} \in K, 1 \leq i \leq s\right\}, \quad d_{j}(x)=\sum\left\{a_{i} d_{j}\left(b p^{\prime}\right)\right\} .
$$

If $p^{r} \nmid j$, then by (2.2) $d_{j}(x)=0 \in L$. If $p^{r} \mid j$, then $d_{j}(x)=\sum\left\{a_{i}\left(d_{j / p^{r}}\left(b_{i}\right)\right)^{p^{r}}\right\}$ $\in F\left(K^{p^{\prime}}\right)=L$. Since $d_{j}$ was arbitrary, $L$ is invariant under $H_{F}^{t}(K)$.

Conversely, assume $L$ is invariant under $H_{F}^{t}(K)$. Assume $L \subseteq F\left(K^{p^{\prime}}\right)$ and $L \nsubseteq F\left(K^{p+1}\right)$ (otherwise $L=F=F\left(K^{p^{n}}\right)$ ). Let $x \in L \backslash F\left(K^{p^{r+1}}\right)$, and let $A$ be a standard generating set for $H_{F}^{t}(K)$. In view of (2.5)c, there exists $d^{i, j} \in A$ such that $d_{z_{i, j} p^{\prime}}^{i, j}(x) \neq 0$. For any $a \in K$, ad $d^{i, j}$ has $z_{i, j} p^{r}$ map $a^{p^{r}} d_{z_{1, j}, p^{r}}^{i, j}$ Since $L$ is invariant under $H_{F}^{t}(K)$, for any $a \in K, a^{p^{r}} d_{i, j p^{\prime}}^{i, j}(x) \in L$. Thus $K^{p^{\prime}} \subseteq L$ and thus $L=F\left(K^{p^{r}}\right)$.

A subfield $L$ of $K$ containing $F$ will be called Galois if $K$ is modular over $L$, i.e., $L$ is the field of constants of a group of rank $t$ higher derivation on $K$ over $F$. We now wish to determine which Galois intermediate fields $L$ have the property that every rank $t$ higher derivation on $L$ over $F$ can be extended to $K$. We will need the following result.

(3.2) Theorem [6, Proposition 3.3, p. 94]. Let $K \supseteq L \supseteq F$ be fields and assume $K$ is modular over $L$ of exponent $e$. The following conditions are equivalent.

(1) There exists an intermediate field $J$ of $K / F$ such that $K=L \otimes_{F} J$ and $J / F$ is modular.

(2) There exists a subbase $B=B_{1} \cup \cdots \cup \cup^{\prime} B_{e}$ of $K$ over $L$ such that $B P^{\prime}$ $\subseteq\left(K^{p^{i}} \cap F\right)\left(\left(L\left(B_{i+1}, \ldots, B_{n}\right)\right) p^{p^{\prime}}\right)$.

(3.3) Lemma. Let $L$ be a subfield of $K$ containing $F$, and assume $L$ is modular over $F$ and that every rank $t$ higher derivation on $L$ over $F$ can be extended to $K$. Let $x \in K$ such that $x^{p^{j}} \in F\left(L^{p^{i}}\right)$. Then $x^{p^{i}} \in\left(K^{p^{i}} \cap F\right)\left(L^{p^{\prime}}\right)$.

Proof. If $x^{p^{i}} \in F$, the result is obvious. Hence assume $x^{p^{i}} \in F\left(L^{p^{\prime}}\right) \backslash F\left(L^{p^{p+1}}\right)$, 
$r \geq i$. Let $T=\left\{x_{i, e} \mid 1 \leq i \leq n, 1 \leq e \leq j_{i}\right\}$ be a subbase for $L$ over $F$, and let $A$ have $T$ as dual base. Write

$$
x^{p^{\prime}}=\sum_{s=1}^{m} a_{s}\left(x_{r+1,1}^{p^{\prime}}\right)^{t_{s,+1,1}} \cdots\left(x_{n, j_{n}}^{p^{\prime}}\right)^{t_{s, n,},}
$$

where $a_{s} \in F, 0 \leq t_{s, j, \ell}<p^{j-r}$, and at least one $t_{s, i, e}$ is not divisible by $p$ (see (2.5)b).

To show $x^{p^{i}} \in\left(K^{p^{i}} \cap F\right)\left(L^{p^{i}}\right)$ it suffices to show each $a_{i} \in K^{p^{i}}$. Proof is by induction on $m$. If $m=1, a_{1} \in K^{p^{l}}$. Assume the result for $m-1$. By induction it suffices to show some $a_{i}$ is in $K^{p}$. Since every higher derivation on $L$ over $F$ can be extended to $K$, and $K^{p}$ is invariant under all higher derivations on $K(2.2)$, any map in any higher derivation on $L$ over $F$ must map $x^{p^{l}}$ into $K^{p^{\prime}}$. We will show some $a_{s}$ is in $K^{p^{i}}$ by induction on the total exponent of (*) (i.e., $\left.\sum t_{s, \alpha, \beta}\right)$. If the total exponent is 1 , then $m=1$ and the result follows. Since $x^{p^{\prime}} \in F\left(L^{p^{\prime}}\right) \backslash F\left(L^{p^{r+1}}\right)$, in view of $(2.5)$ c, some $d_{z_{i, e} p^{\prime}}^{i, e}\left(x^{p^{t}}\right) \neq 0$. Applying $d_{z_{i, e} p^{\prime}}^{i, e}$ to (*) yields a nonzero element of $K^{p^{\prime}}$ of lower total exponent with nonzero coefficients of the form $w a_{s}, w \in Z /(p)$. If $d_{z_{i, e}}^{i, e}\left(x^{p^{\prime}}\right) \notin F$, then by induction some $w a_{s}$, hence some $a_{s}$, is in $K^{p^{t}}$ and the result follows. If $d_{z_{i \in} p^{\prime}}^{i, e}\left(x^{p^{t}}\right) \in F$, then since

$$
\left(x_{r+1,1}^{p^{r}}\right)^{t_{s,+1,1}} \cdots\left(x_{n, j_{n}}^{p^{r}}\right)^{t_{s, j_{n},},} \quad 0 \leq t_{s, i, e}<p^{i-r}
$$

is a vector space basis for $F\left(L^{p^{\prime}}\right)$ over $F$, in view of $(2.5) \mathrm{d}, d_{z_{i, b} p^{\prime}}^{i, e}\left(x^{p^{\prime}}\right)=a_{s}$ for some $s$. Thus once again some $a_{s}$ is in $K^{p^{i}}$ and the result is established.

(3.4) Theorem. Let $L$ be a Galois subfield of $K$ containing $F$ and assume $L$ is modular over $F$. Then every rank $t$ higher derivation on $L$ over $F$ extends to $K$ if and only if there exists a field $J, K \supseteq J \supseteq F, J$ is modular over $F$ and $K=L \otimes_{F} J$.

Proof. If $K=L \otimes_{F} J$, then every rank $t$ higher derivation on $L$ over $F$ can be extended to $K$ by acting trivially on $J$.

Assume now that every rank $t$ higher derivation on $L$ over $F$ can be extended to $K$. Let $B=B_{1} \cup \cdots \cup B_{n}$ be a subbase for $K$ over $L$ where $B_{i}$ is of exponent $i$ over $L$. We claim $B_{r}^{p^{\prime}} \subseteq F\left(L^{p^{\prime}}\right)$. Let $A$ be a standard set of generators of $H_{F}^{t}(L)$ with dual basis $\left\{x_{i, e} \mid 1 \leq i \leq n, 1 \leq e \leq j_{i}\right\}$. In view of $(2.5)$ c, $F\left(L^{p^{r}}\right)$ is the field of constants of the set of maps $S=\left\{d_{z_{i, p} p^{i,}}^{i} \mid 1 \leq i \leq n, 1 \leq e \leq j_{i}\right.$, $\left.0 \leq c_{i}<\min (i, r)\right\}$. Thus it suffices to show $x^{p^{r}}$ is annihilated by all maps in $S$. If $p \nmid z_{i, e}$ since $d^{i, e}$ can be extended to $K$,

$$
d_{z_{i, e} p^{c_{i}}}^{i, e}\left(x^{p^{r}}\right)=0, \quad 0 \leqslant c_{i}<\min (i, r)
$$

(2.2). If $p \mid z_{i, e}$ consider $V\left(d^{i, e}\right)$ (see $\S I$ II). We claim $\left(z_{i, e}+1\right) p^{c_{i}} \leq t$ if $0 \leq c_{i}$ $<\min (i, r)$ (unless $t=1$, in which case the result is obvious). For if not, $\left(z_{i, e}+1\right) p^{i-1}>t$, hence $z_{i, e}+1>t / p^{i-1}$ and $z_{i, e / p}+1 / p>t / p^{i}$, a contradiction to the definition of $z_{i, e}(2.4)$. Since $p \nmid\left(z_{i, e}+1\right)$, we see again $d_{z_{i, e} p^{c i}}^{i, e}\left(x^{p^{\prime}}\right)=0,0$ $\leq c_{i}<\min (i, r)$. Thus $x^{p^{r}} \in F\left(L^{p^{\prime}}\right)$, and $B_{r}^{p^{\prime}} \subseteq F\left(L^{p^{\prime}}\right)$. By Lemma 3.3, 


$$
B_{r}^{p^{\prime}} \subseteq\left(K^{p^{\prime}} \cap F\right)\left(L^{p^{\prime}}\right) \subseteq\left(K^{p^{\prime}} \cap F\right)\left(\left(L\left(B_{r+1}, \ldots, B_{n}\right)\right)^{p^{\prime}}\right) .
$$

The result now follows from (3.2).

(3.5) Corollary. Let $L$ be a Galois subfield of $K$ containing $F$. Let $M$ be the field of constants of all rank $t$ higher derivations on $L$ over $F$. Then every rank $t$ higher derivation on $L$ over $F$ extends to $K$ if and only if there exists a field $J, K \supseteq J \supseteq M$, $J$ is modular over $M$ and $K=L \otimes_{M} J$.

Proof. Since $K$ is modular over $L$, and every rank $t$ higher derivation on $L$ over $F$ extends to $K, K$ is modular over $M$. Applying (3.4) to the chain of fields $K \supseteq L \supseteq M$ yields the result.

(3.6) Corollary. Let $L$ be a subfield of $K$ containing $F$. Then $L$ is invariant under $H_{F}^{t}(K)$ and every rank $t$ higher derivation on $L$ over $F$ extends to $K$ if and only if $L=F$ or $L=K$.

Proof. Apply (3.1) and (3.5), (3.1) showing $K$ is modular over $L$.

IV. An intermediate theory. Assume $E$ is a normal separable extension of $H$. In classical Galois theory, the distinguished intermediate subfields $D$ are characterized by being invariant under the group of automorphisms of $E$ over $H$. They also possess the property that the Galois group of $E$ over $H$ when restricted to $D$ is the Galois group of $D$ over $H$.

As usual, let $K$ be a finite dimensional purely inseparable modular extension of $F$ and let $L$ be an intermediate field such that $K$ is modular over $L$. In view of (3.1) the requirement that $L$ be invariant under $H_{F}^{t}(K)$ is much too restrictive. Considering the Dedekind independence theorem for automorphisms, (2.5)c, and [7, Theorem 19, p. 186], a natural alternative to this condition is that there exists a standard generating set for $H_{F}^{t}(K)$ which leaves $L$ invariant. As we shall see, this invariance condition gives rise to an interesting intermediate theory. We observe that if $L$ is invariant under a standard generating set for $H_{F}^{t}(K)$, then the restriction of that set to $L$ will have field of constants $F$, and hence $L$ must be modular over $F$. Moreover, since the higher derivations $d^{i, j}$ in a standard for $H_{F}^{t}(K)$ are iterative, $\left.d^{i, e}\right|_{L}$ we will have first nonzero map $d_{z_{i, j} p^{p}}$ for some $r$, and will be iterative of index $z_{i, j} p^{r}$. In this section we will characterize such intermediate fields $L$.

(4.1) Definition. Let $L$ be a Galois subfield of $K$ containing $F$. Then $L$ is distinguished if and only if there exists a standard generating set for $H_{F}^{t}(K)$ which leaves $L$ invariant.

We will begin by examining the simplest type of modular extension. Recall $[K: F]<\infty$.

(4.2) Definition. Let $K$ be a modular extension of $F$. Then $K$ is an equiexponential modular extension of $F$ if and only if there exists a subbase $\left\{x_{1}, \ldots, x_{n}\right\}$ for $K$ over $F$ such that each $x_{i}$ has exponent $r$ over $F$ for some fixed integer $r$. 
(4.3) Lemma. A modular extension $K$ of $F$ is equiexponential if and only if every relative p-base for $K$ over $F$ is also a subbase for $K$ over $F$.

Proof. If $K$ is an equiexponential modular extension of $F$, then since a subbase is a relative $p$-base of minimal total exponent, any relative $p$-base will be a subbase. Conversely, assume $K$ is not equiexponential over $F$ and let $\left\{x_{1,1}, \ldots\right.$, $\left.x_{n, j_{n}}\right\}$ be a subbase. Then $\left\{x_{1,1}-x_{n, j_{n}}, \ldots, x_{n, j_{n-1}}-x_{n, j_{n}}, x_{n, j_{n}}\right\}$ is a relative $p$-base for $K$ over $F$, and is not of minimal total exponent, and hence is not a subbase for $K$ over $F$.

(4.4) Theorem. Assume $K$ is an equiexponential modular extension of $F$. If $L$ is an intermediate such that $K$ is modular over $L$, then $L$ is modular over $F$.

Proof. Let the exponent of $K$ over $F$ be $n$, and let $\left\{x_{1}, \ldots, x_{s}\right\}$ be a subbase for $K$ over $L$. By (2.3), there exists a set $T \subset L$ such that $T \cup\left\{x_{1}, \ldots, x_{s}\right\}$ is a $p$-basis for $K$. Since $\left\{x_{1}, \ldots, x_{s}\right\}$ is relatively $p$-independent in $K$ over $L$, it is relatively $p$ independent in $K$ over $F$. Thus $\left\{x_{1}, \ldots, x_{s}\right\}$ can be completed to a relative $p$-basis for $K$ over $F$ with elements from $T$. Let $\left\{x_{1}, \ldots, x_{s}, Y_{1}, \ldots, Y_{r}\right\}$ be such a relative $p$-basis. Since $K$ is equiexponential modular over $F,\left\{x_{1}, \ldots, x_{s}, Y_{1}, \ldots, Y_{r}\right\}$ is also a subbase for $K$ over $F$. Let $\left\{x_{1}, \ldots, x_{t}\right\}$ be of exponent $n$ over $L$, and let $x_{j}$ be of exponent $e_{j}$ over $L, t+1 \leq j \leq s$. By a degree argument, we observe $L$ $=F\left(x_{i+1}^{p_{r+1}}, \ldots, x_{s}^{p^{s_{1}}}, Y_{1}, \ldots, Y_{r}\right)$, and since $\left\{x_{1}, \ldots, x_{s}, Y_{1}, \ldots, Y_{r}\right\}$ is a subbase for $K$ over $F,\left\{x_{i+1}^{p_{i+1}}, \ldots, Y_{r}\right\}$ is a subbase for $L$ over $F$. Thus $L$ is modular over $F$.

(4.5) Corollary. Assume $K$ is an equiexponential modular extension of $F$, and $L$ is an intermediate field such that $K$ is modular over $L$. Then there exists a subbase $\left\{x_{1}, \ldots, x_{n}\right\}$ for $K$ over $F$ such that $\left\{x_{s}^{p^{e n}}, \ldots, x_{n}^{p_{n}}\right\}$ is a subbase for $L$ over $F$ for some $e_{s}, \ldots, e_{n}$.

(4.6) Example. The converse of Theorem (4.4) does not hold. Consider the following chain of fields where $P$ is a perfect field (char $P \neq 0$ ), and $x, y, z$ are algebraically independent over $P$.

$$
K=P\left(x, y^{p} x+z^{p}, z^{p^{2}}\right), \quad L=P\left(x^{p^{2}}, y^{p^{2}}, z^{p^{2}}\right), \quad F=P\left(x^{p^{2}}, y^{p^{3}}, z^{p^{2}}\right) .
$$

Elementary calculations show $K$ is equiexponential modular over $F$, and $L$ is modular over $F$. However, $K$ is not modular over $L$.

(4.7) Definition. Let $K$ be a modular extension of $F$, and let $L$ be a Galois intermediate field. Then $L$ is homogeneous with respect to $K$ if and only if there exists $T=T_{1} \cup \cdots \cup T_{n}$ a subbase for $K$ over $F$ where $F\left(T_{i}\right)$ is equiexponential modular over $F$, and such that $L=L \cap F\left(T_{1}\right) \otimes \cdots \otimes L \cap F\left(T_{n}\right)$.

(4.8) Proposition. Let $K$ be a modular extension of $F$ and let $L$ be a homogeneous intermediate field. Then $L$ is modular over $F$ if and only if $L \cap F\left(T_{i}\right)$ is modular over $F$ for all $i$.

Proof. Assume $L$ is modular over $F$, and let $\left(L \cap F\left(T_{i}\right)\right)^{*}$ be the unique 
minimal extension of $L \cap F\left(T_{i}\right)$ which is modular over $F$. Since both $L$ and $F\left(T_{i}\right)$ are modular over $F,\left(L \cap F\left(T_{i}\right)\right)^{*} \subseteq L \cap F\left(T_{i}\right)$, and hence $\left(L \cap F\left(T_{i}\right)\right)^{*}=L$ $\cap F\left(T_{i}\right)$. The converse follows since a tensor product of tensor products is a tensor product.

(4.9) Definition. Let $K$ be a modular extension of $F$, and let $L$ be an intermediate field. Then $L$ is $M$-homogeneous if and only if there exists a subbase $T=T_{1} \cup \cdots \cup T_{n}$ of $K$ over $F$ such that $L=L \cap F\left(T_{1}\right) \otimes \cdots \otimes L \cap F\left(T_{n}\right)$ and $F\left(T_{i}\right)$ is modular over $L \cap F\left(T_{i}\right)$ for all $i$.

(4.10) Example. $L$ may be $M$-homogeneous for some subbases of $K$ over $F$, and only homogeneous for others. Let $P$ be a perfect field, and $x, y, z$ algebraically independent over $P$. In the following diagram with $T_{1}=\left\{z^{p}-x^{p}\right\}$ and $T_{2}$ $=\left\{x, y^{p} x+z^{p}\right\}, L$ is homogeneous but $F\left(T_{2}\right)$ is not modular over $L \cap F\left(T_{2}\right)$.

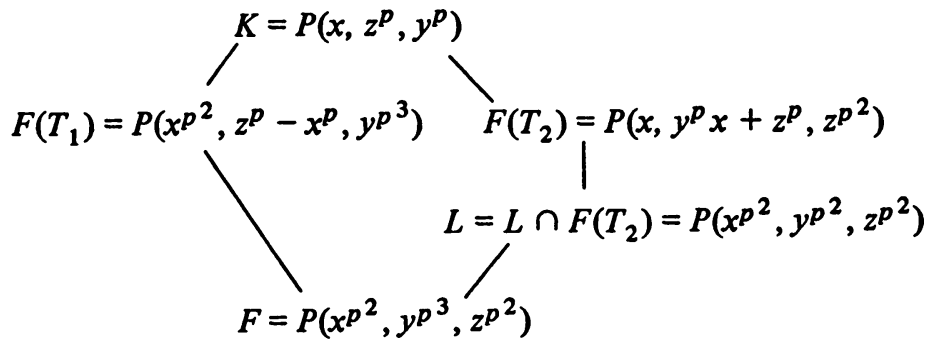

However, if we set $T_{1}=\left\{z^{p}\right\}$ and $T_{2}=\left\{x, y^{p}\right\}$, the following diagram shows $L$ is $M$-homogeneous.

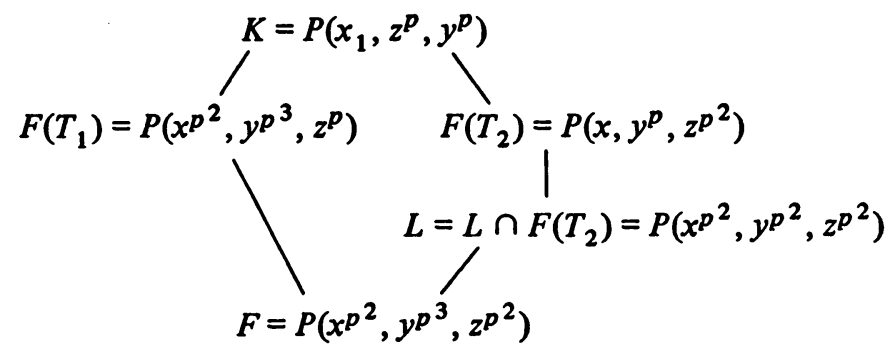

Conjecture. $L$ is homogeneous if and only if $L$ is $M$-homogeneous.

(4.11) Proposition. Let $K$ be a modular extension of $F$, and let $L$ be an $M$ homogeneous intermediate field. Then $L$ is also modular over $F$.

Proof. By assumption, $F\left(T_{i}\right)$ is modular over $L \cap F\left(T_{i}\right)$ for all $i$. By (4.4), $L \cap F\left(T_{i}\right)$ is modular over $F$ for all $i$, and hence $L$ is modular over $F(4.8)$.

(4.12) Theorem. Assume $K$ is a modular extension of $F$ and $L$ is a Galois intermediate field. Then $L$ is distinguished if and only if $L$ is $M$-homogeneous.

Proof. Assume $L$ is distinguished and let $A$ be a standard generating set for $H_{F}^{t}(K)$ which leaves $L$ invariant. Let $T=T_{1} \cup \cdots \cup T_{n}$ be a dual base for $A$. 
We claim $L=L \cap F\left(T_{1}\right) \otimes \cdots \otimes L \cap F\left(T_{n}\right)$. Let $A=A_{1} \cup \cdots \cup A_{n}$ where

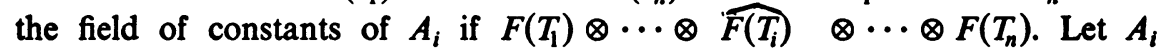
$=\left\{d^{i, 1}, \ldots, d^{i, j_{i}}\right\}$ and let $d^{i, s} \mid L$ have first nonzero map $r_{i, s}$. Let $\overline{A_{i}}=\left\{d_{c}^{i, s} \mid 1 \leq s\right.$ $\left.\leq j_{i}, 0 \leq c<r_{i, s}\right\}$. Then the field of constants of $\overline{A_{i}}$ is of the form $F\left(T_{1}\right) \otimes \cdots$ $\otimes H_{i} \otimes \cdots \otimes F\left(T_{n}\right)$. Since $L$ is the field of constants of $\cup \bar{A}_{i}$,

$$
L=\cap\left\{F\left(T_{1}\right) \otimes \cdots \otimes H_{i} \otimes \cdots \otimes F\left(T_{n}\right) \mid 1 \leq i \leq n\right\}=H_{1} \otimes \cdots \otimes H_{n} .
$$

Thus $L$ is homogeneous and since $\bar{A}_{i} I_{F\left(T_{i}\right)}$ has $H_{i}$ as field of constants, $L$ is $M$ homogeneous.

Conversely, assume $L$ is $M$-homogeneous and let $L=L_{1} \otimes \cdots \otimes L_{n}$. Then since $F\left(T_{i}\right)$ is modular over $L_{i}$, and $F\left(T_{i}\right)$ is equiexponential modular over $F$, in

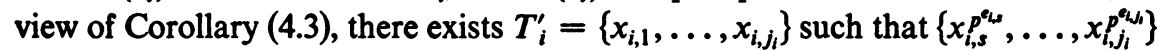
(possibly renumbering) is a subbase for $L_{i}$ over $F$. Thus if we let $A$ be the standard generating set for $H_{F}^{t}(K)$ with $T_{i}^{\prime}$ as dual basis, elementary calculations show $L$ is invariant under $A$.

(4.13) Corollary. Assume $K$ is a modular extension of $F$, and $L$ is a Galois subfield. The following are equivalent.

(1) $L$ is distinguished.

(2) $L$ is $M$-homogeneous.

(3) There exists a subbase $\left\{x_{1}, \ldots, x_{n}\right\}$ for $K$ over $F$ such that $\left\{x_{i}^{p^{a_{1}}}, \ldots, x_{n}^{p_{n}}\right\}$ is a subbase for $L$ over $F$ for some $e_{1}, \ldots, e_{n}$.

We have seen (4.11) that the class of distinguished intermediate fields is contained in $\{L \mid K$ is modular over $L$ and $L$ is modular over $F\}$. We close with an example to show this containment may be proper.

Let $P$ be a perfect field (char $P \neq 0$ ) and let $x, y$ be algebraically independent over $P$. Consider the following chain of fields.

$$
K=P(x, y), \quad L=P\left(x-y^{p^{2}}, x^{p}\right), \quad F=P\left(x^{p}, y^{p^{4}}\right) .
$$

Elementary calculations show all extensions are modular. Since $K$ is generated over $L$ by a single element $y$, if $L$ is homogeneous, then $K=L \otimes_{F} L^{\prime}$ for some $L^{\prime}$. But since $L$ is of exponent 2 over $F$, this is impossible. Thus $L$ is not homogeneous, and hence not distinguished.

\section{REFERENCES}

1. N. Heerema, Convergent higher derivations on local rings, Trans. Amer. Math. Soc. 132 (1968), 31-44. MR 36 \#6406.

2.—, Derivations and embeddings of a field in its power series ring. II, Michigan Math. J. 8 (1961), 129-134. MR 25 \#69.

3. F. Zerla, Iterative higher derivations in fields of prime characteristic, Michigan Math. J. 15 (1968), 407-415. MR 39 \# 185.

4. N. Heerema and J. Deveney, Galois theory for fields $K / k$ finitely generated, Trans. Amer. Math. Soc. 189 (1974), 263-274. 
5. R. Davis, A Galois theory for a class of purely inseparable field extensions, Dissertation, Florida State University, Tallahassee, Fla.

6. J. N. Mordeson and B. Vinograde, Structure of arbitrary purely inseparable extension fields, Lecture Notes in Math., vol. 173, Springer-Verlag, Berlin and New York, 1970. MR 43 \# 1952.

7. N. Jacobson, Lectures in abstract algebra. Vol. III: Theory of fields and Galois theory, Van Nostrand, Princeton, N. J., 1964. MR 30 \#3087.

Department of Mathematics, Florida State University, Tallahassee, Florida 32306

Current address: Department of Mathematics, Kansas State University, Manhattan, Kansas 66502 